\title{
A FIRST APPROXIMATION TO SIMULATE THE ELECTRO-POLYMERIZATION PROCESS
}

\author{
M. B. CAMARADA ${ }^{a}$, M.C. GIMÉNEZ ${ }^{c}$,W. SCHMICKLER, M. A. DEL VALLEa* \\ ${ }^{a}$ Pontificia Universidad Católica Chile, Facultad de Química, Lab. Electroquímica de Polímeros, Av. Vicuña Mackenna 4860, Santiago, Chile \\ ${ }^{a}$ Department of Theoretical Chemistry, Ulm University, D-89069 Ulm, Germany \\ 'IFEG, FaMAF, Universidad Nacional de Córdoba, Córdoba, Argentina \\ (Received: January 5, 2012 - Accepted: May 10, 2012)
}

\begin{abstract}
With the aim of understanding the nucleation and growth mechanism of thiophene, a new computational simulation method based on a kinetic Monte Carlo algorithm was designed. It reproduces key processes such as diffusion, oligomerization, and the precipitation of oligomers onto the electrode surface. This paper describes all simulation details, reports the first computational results and contrasts them with previously published electro-polymerization evidence. The results agree well with experimental studies and demonstrate how computational simulations can help to understand the electrochemical process of conducting polymers formation.
\end{abstract}

Keywords: kinetic Monte Carlo, conducting polymers, thiophene, electro-polymerization

\section{INTRODUCTION}

In 1977 Shirakawa et al. ${ }^{1}$ reported significant increases in the conductivity of poly(acetylene) upon halogen doping. Since then, the field of conducting polymers, also called synthetic metals, has aroused great technological and scientific interest.

In the last two decades, second generation polymers such as poly(pyrrol $)^{2}$, poly(furan $)^{3-5}$, poly(thiophene $)^{6,7,42}$ and their derivatives, have provoked great attention because of their remarkable electrical and optical features. Oligo and poly(thiophene) have attracted particular consideration due to their high processability and excellent environmental stability. Their applications are wide-ranging and include surface light emitting diodes (SLED's) ${ }^{8}$, light emitting diodes (LED's) s.15 $^{9}$, photovoltaic cells ${ }^{16-20}$ and transistors ${ }^{21-27}$.

These $\pi$-conjugated compounds can be synthesized by chemical or electrochemical means. Electro-polymerization presents several advantages compared to other methods, in particular the absence of catalyst other than the electrode, direct grafting of the doped film onto the electrode surface, in situ characterization by different techniques and easy control of the deposit properties $^{28}$. The anodic route of synthesis is a unique process, which is initiated by the oxidation of a neutral monomer at the anode surface producing radical cation species that react to form longer oligomeric chains ${ }^{29}$. The electrochemical synthesis of conducting polymers is a very complex process where many experimental factors are involved such as the chemical nature and concentration of monomer and electrolyte, the solvent, the physical and chemical nature of the electrode substrate, and the conditions of the electrical perturbation applied to the interface. In the literature, we can find several experimental studies about the nucleation and growth mechanism of conducting polymers; however, there are still unsolved questions regarding the exact mechanism. A detailed process comprehension would provide the necessary tools for controlling the structure and intrinsic properties of the film a priori, useful for the design of new structures with improved properties.

Because of the high complexity of the electro-polymerization, theoretical electrochemical treatments by ab initio methods are impossible ${ }^{30}$. However, computational simulations can provide information that is experimentally inaccessible ${ }^{31,32}$. Simulations of electrochemical processes at realistic time require stochastic methods such as kinetic Monte Carlo, which by necessity give less detailed information than deterministic methods.

With the aim of understanding the nucleation and growth mechanism of thiophene (Th) we have designed a new computational simulation method based on a kinetic Monte Carlo algorithm. It reproduces key processes such as diffusion, oligomerization, and the precipitation of oligomers onto the electrode surface. In this paper we describe all simulation details, report the first computational results and contrast them with previously published electropolymerization evidence. The results agree well with experimental studies and demonstrate how computational simulations can help to understand the electrochemical process of conducting polymers formation.

\section{Model and simulation method}

\subsection{The oligomerization process}

Electro-polymerization starts with the diffusion of monomers toward the electrode surface, where an electron is transferred from the monomer to the working electrode, which has a high enough potential to overcome the energetic barrier of the process. The dimer is produced from the union of two oxidized species (monomeric radical cations), the trimer from the combination of one activated dimer with a monomeric cation radical. In this way, longer oligomeric chains are formed and collected in a high density oligomeric region $(\mathrm{HDOR})^{33}$. When the oligomerization process has set in and supersaturation is attained, oligomers are deposited on the electrode surface as clusters, giving place a nucleation and growth process. The precipitation occurs due the insolubility of some oligomers that reach the critical chain length, the limit length of oligomeric chains at which precipitation begins.

\subsection{Simulation details}

A Fortran77 code was developed to model an electrode surface in contact with an electrolyte solution that initially contains monomers, molecules of solvent and the supporting electrolyte. Within the code, the monomers are represented as spheres; because of size restriction, the electrolyte is not modeled as individual molecules but as a heat bath. We used a three dimensional reticular model (Figure 1) with a total of $[\mathrm{s} \cdot \mathrm{s} \cdot(\mathrm{h}+\mathrm{r})]$ positions, each representing a cube with an edge length $d$. The size of $d$ was selected according to the crystallographic center-center distance of the rings of bithiophene $e^{34}$ $(d=3.89 \AA)$. The simulation cell contains at the upper end a reservoir of monomers, labeled A in Figure 1, with a constant number of particles. During the course of the simulation particles were added to $\mathrm{A}$ as needed. A region $\mathrm{B}$, the reaction zone, is delimited by region $\mathrm{A}$ at the top and at the bottom by a box representing the working electrode. Periodic boundary conditions were implemented along the code in the $\mathrm{x}$ and $\mathrm{y}$ directions parallel to the electrode surface. The reservoir must be placed at a distance sufficiently large so that the monomers in A do not interact with particles near the surface. Particle entrance from zone B to A was not allowed.

Based on an experimental study of thiophene ${ }^{35}(\mathrm{Th})$, we took the critical chain length as three units. Thus, oligomers with three or more units of length will precipitate on the electrode surface.

The simulation starts with the diffusion of monomers from the reservoir to the electrode, and follows the rules of kinetic Monte Carlo. Every movement is selected randomly, and its probability is directly proportional to the magnitude of its rate in the processes catalogue, i.e. higher rate values have greater probabilities of being selected. The time corresponding to a movement $\mathrm{G}$ is calculated from a randomly chosen number $\mathrm{m}$, selected by a code's subroutine ${ }^{41}$, uniformly distributed between 0 and 1 according to Eq. 1 :

$$
\Gamma=-\frac{1}{R} \ln (\mu)
$$




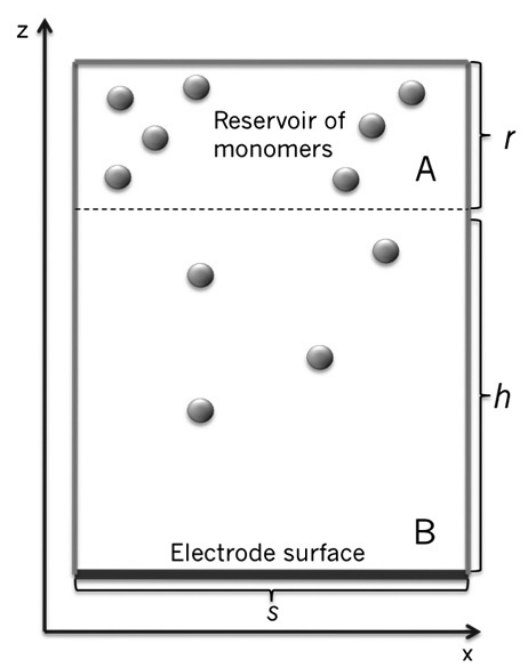

Figure 1: Schematic representation of the simulation box.

where $R$ corresponds to the total sum of all rates included in the processes catalogue.

When a monomer reaches the first layer of the box or gets in contact with already deposited oligomers, is automatically labeled as a radical cation, and can diffuse inside the zone B again. This radical monomer can react with other activated monomer as depicted in Figure 2a creating a neutral dimer.

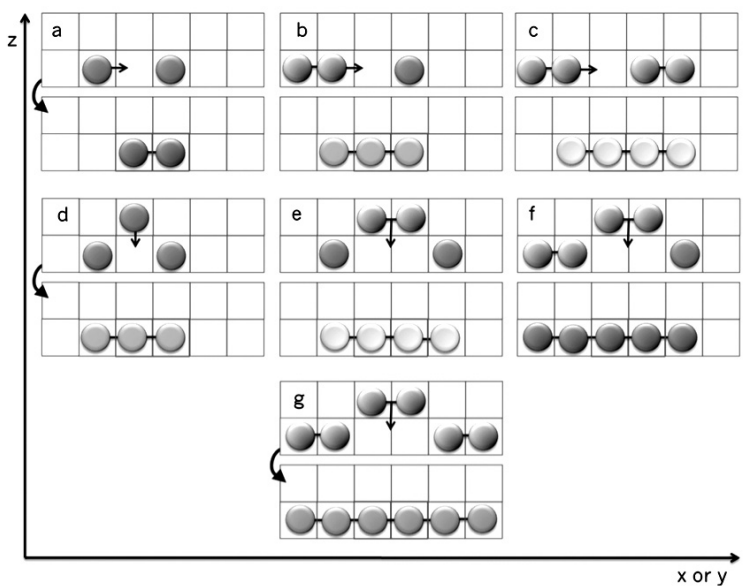

Figure 2: The different thiophene oligomers formed during the simulation. Each case is explained within the main text

Dimers can also become radical species in the same way as monomers, and can react with cationic monomers or dimers producing neutral trimers and tetramers, respectively (Figure 2b,c). Reactions between radicals satisfied two different criteria:

1. In accordance with experimental evidence, which indicates that the film grows parallel to the metal surface ${ }^{36}$, the generation of oligomeric chains with geometry parallel to the $\mathrm{z}$ axis was not allowed. In a real system the generation of oligomers vertical to the surface occurs, however, the rotation of this kind of structures was not implemented with the aim of simplifying the computational code. This consideration will be reflected in a lower production of trimers, tetramers, pentamers and hexamers.

2. The formation of branched or nonlinear chains was not permitted, in order to preserve the planarity and linearity of the oligomers.

Triple linkage can also occur when radicals move up or down in the $\mathrm{z}$ axis and find two activated oligomers lined up on the electrode surface, generating trimers, tetramers, pentamers and hexamers (Figure 2d-g).

When the size of the chain exceeds two units, the oligomer can still diffuse inside the zone $\mathrm{B}$, however, upward movements in the $\mathrm{z}$ direction are not allowed to guarantee the precipitation on the electrode. When insoluble oligomers reach the bottom of the simulation box or touch already deposited oligomers they become immediately fixed and thus, dendritic growth is expected. The diffusion of precipitated species is not permitted.

The size of the simulation box was established taking into account the typical monomer concentration in an experimental procedure, $0.01 \mathrm{M}$. For the electrode surface [28x28] cubic unit cells (of side length $3.89 \AA$ ) were defined. The height of zone A was chosen as 14 unit cells, and the height of zone B was optimized as follows: we selected different heights $(h)$ for zone B and ran simulations at the same concentration and with the same size of reservoir ([28x28x14]) till the deposit crossed $h / 2$. The coverage (number of occupied sites per layer) was calculated for each simulation at the final step. Plots of the coverage of the deposit against $h$ at the end of each simulation step were fitted using Eq.2:

$$
f(h)=C \cdot \operatorname{erfc}\left[\frac{\left(h-h_{0}\right)}{L}\right]
$$

where erfc denotes the complementof the error function. Table 1 summarizes the fitting parameters $C, h_{0}$ and $L$ and the root-mean-square deviation (RMSD). RMSD values remain almost constant from $h=56$ onwards.

Table 1: Height of zone B (in unit cells), fitting parameters $C, h_{0}$ and $L$ and the root-mean-square deviation (RMSD) using Eq. 2.

\begin{tabular}{|c|c|c|c|c|}
\hline$h$ & $C$ & $h_{0}$ & $\mathrm{~L}$ & RMSD \\
\hline 18 & 0.103 & 6.26 & 3.059 & 0.16612 \\
\hline 28 & 0.089 & 12.64 & 2.801 & 0.02787 \\
\hline 36 & 0.108 & 13.48 & 5.600 & 0.01871 \\
\hline 42 & 0.098 & 18.82 & 7.648 & 0.01749 \\
\hline 48 & 0.098 & 19.54 & 5.545 & 0.01856 \\
\hline 56 & 0.113 & 23.03 & 7.001 & 0.01644 \\
\hline 68 & 0.102 & 22.98 & 6.678 & 0.01631 \\
\hline 78 & 0.115 & 23.22 & 7.345 & 0.01656 \\
\hline
\end{tabular}

Therefore, this value of $h$ was taken as the height for zone B. The rate catalogue included diffusion rates for thiophene oligomers from 1Th to $6 \mathrm{Th}$. To reduce computational costs and calculation time, only the rates of the selected oligomer and its neighbors are upgraded in the computational code.

Since this is an exploratory study, all rates were set to $1.00 \mathrm{~nm} / \mathrm{s}$, which means that all processes have the same probability of being chosen; therefore the simulation time has no absolute meaning. In principle, diffusion coefficients for each oligomer (1Th-6Th) in condensed phase at different temperatures can be calculated from Molecular Dynamic simulations through the EinsteinStokes expression. The introduction of these values in to our KMC simulations will be analyzed in a subsequent work.

\section{FIRST RESULTS}

Results are averages over twenty runs. For all calculations a reservoir of [28x28x14] unit cells and a zone B of [28x28x56] unit cells were employed. All simulations finished when the deposit crossed $h / 2$ in zone B; therefore all films have the same height but the simulation time varies depending of the parameters selected.

In this Section, the influence of the concentration on the film growth is analyzed and contrasted with previous experimental studies reported by our group.

In this exploratory study we focused on the effect of the monomer concentration on the structure of the deposited film. A wide range of monomer concentrations was selected $(0.01 \mathrm{M}, 0.1 \mathrm{M}, 0.5 \mathrm{M}$ and $1.0 \mathrm{M})$ to simulate the electro-polymerization process.

According to experimental evidence, at higher monomer concentrations we expect an increase in the number of radical cations ${ }^{37}$. Then, insoluble species will appear faster in the solution, and the nucleation time $\tau$, when the first nucleus appears, should be shorter.

Indeed, as Figure 3 depicts, the number of cationic oligomers grew as the initial concentration increased. Because of the higher number of activated 
oligomers, there was more production of longer oligomers, $3 \mathrm{Th}$ and $4 \mathrm{Th}$, as Figure 4 shows.

a

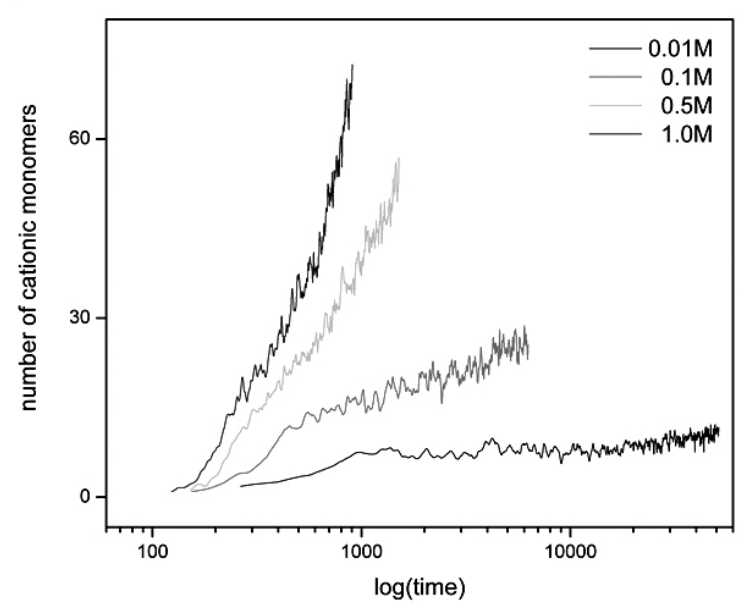

b

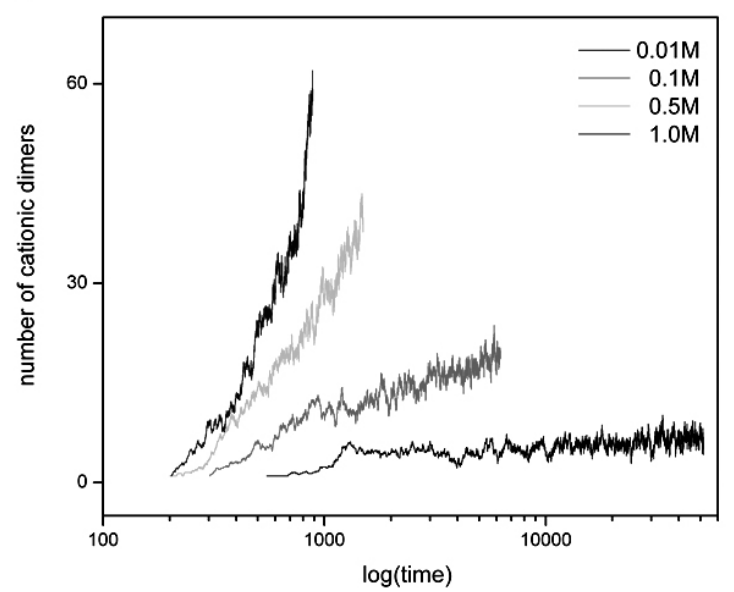

Figure 3: Number of thiophene cationic monomers (a) and dimers (b) during the simulation time in logarithmic scale.

a

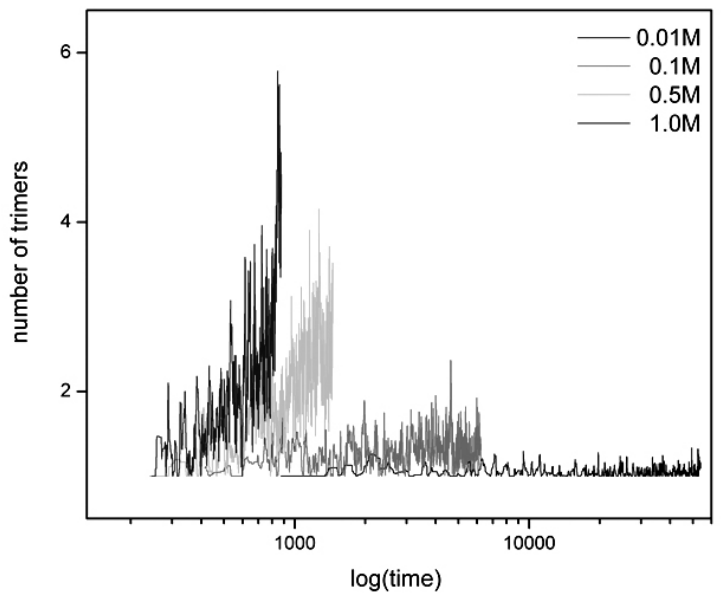

b

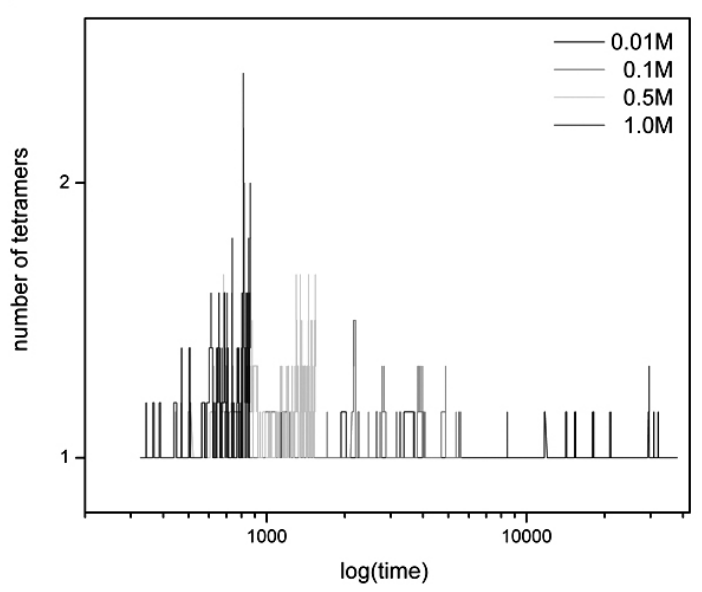

Figure 4: Number of thiophene trimers (a) and tetramers (b) during the simulation time in logarithmic scale

In all runs, the number of 4Th remained small, and chains of five and more units were not observed during these simulations. Probably the introduction of realistic diffusion rates for all involved species will provide a more diverse growth. In all simulations trimers and tetramers diffused into the solution before precipitation, forming a high density oligomeric region (HODR) as observed in experiment.

A plot of the nucleation time $\tau$ against the monomer concentration (Figure 5) gives analogous results to the experimental evidence; $\tau \square \square$ decreases as the concentration grows.

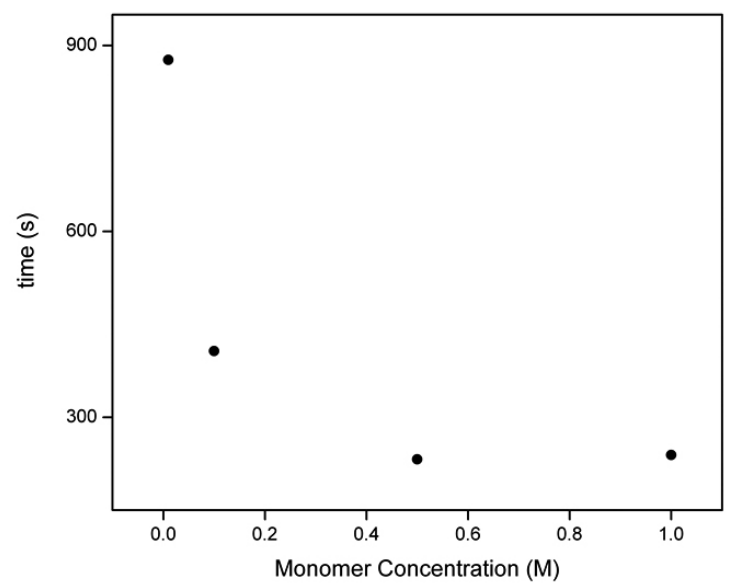

Figure 5: The time at the first oligomer precipitates $(\tau)$ at each monomer concentration.

Schrebler et al..$^{38}$, described the effects of different experimental factors on electro-polymerization. They conclude that as the monomer concentration progressively grows, the deposit mass (at equal deposition times) increase.

Figure 6 illustrates the total number of deposited tiophene units against time. For the sake of comparison, the same final time of simulation was set up for all concentrations. $\tau$ was subtracted from all curves with the aim of having equivalent starting points, i.e. the moment where the first oligomer is deposited. At higher initial monomer concentration, the number of deposited thiophene units increased, as the experimental evidence previously indicated. A larger amount of thiophene units means higher deposited mass. 


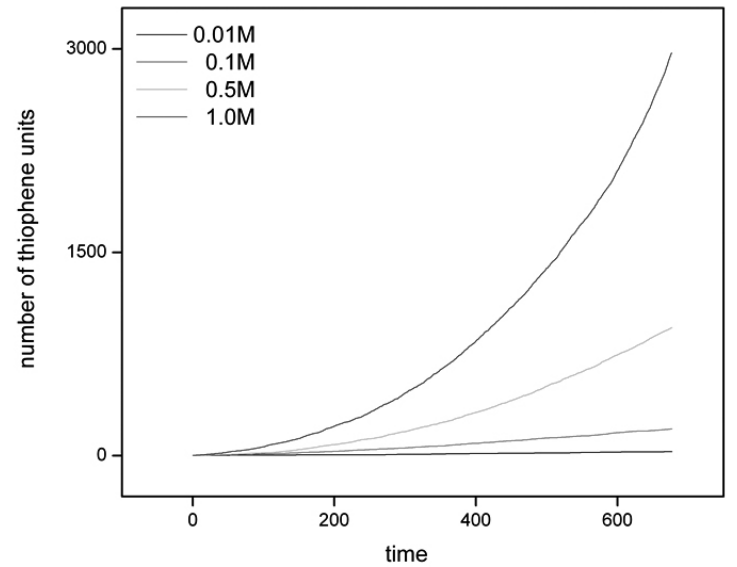

Figure 6: Total number of thiophene units deposited during the simulation till the same final time, at different concentrations

Comparing the percentage of coverage degree (number of occupied sites per layer) against height $(h)$ (Figure 7a) for all concentrations at the end of the simulations (when the film reached a height of $h / 2$ ), it can be seen that as the concentration grows up, the film presents less density of thiophenic units from approximately $h=18$.

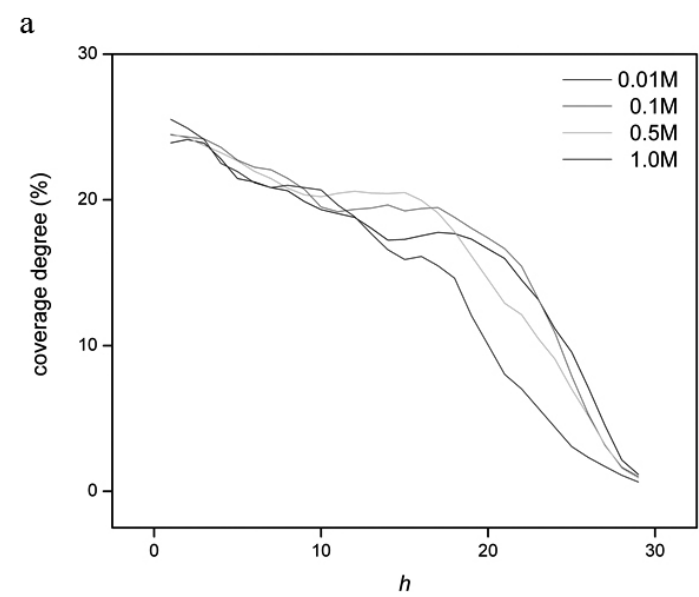

b

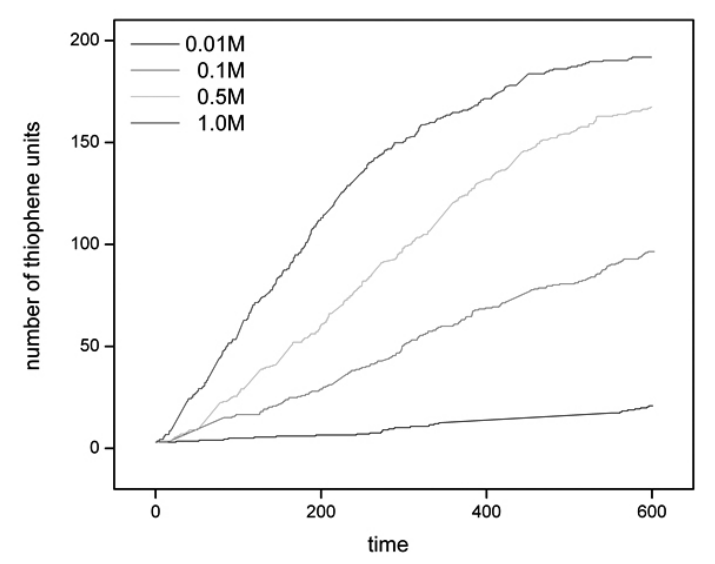

Figure 7: Coverage degree at $t_{f}$ of each layer of the deposit (a) and the number of thiophene units on the electrode surface $v s$ the simulation time (b), at different concentrations
The deposits formed at $0.5 \mathrm{M}$ and $1.0 \mathrm{M}$ are less compact presenting dendritic growth. This can be confirmed comparing the snapshots (Figure 8) obtained for every concentration at the end of the simulation. At higher concentrations more radicals are generated near the surface of the electrode or the just formed conducting polymer. The probabilities of producing longer oligomeric species with short-range diffusion are higher, giving place for a massive and less structured precipitation phenomenon.

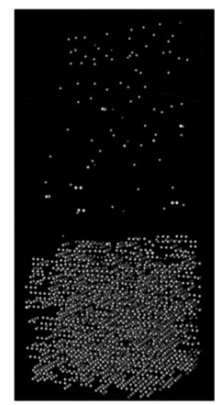

$0.01 \mathrm{M}$

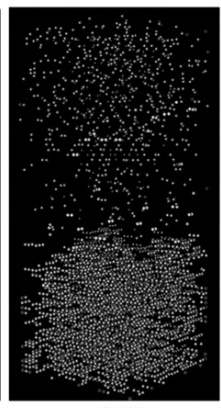

$0.1 \mathrm{M}$

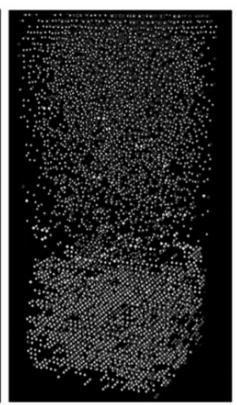

$0.5 \mathrm{M}$

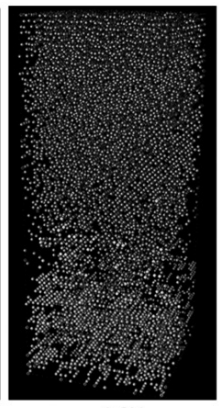

$1.0 \mathrm{M}$
Figure 8: Snapshots of the eletro-polymerization simulation of thiophene at different concentrations

Experimental studies regarding the nucleation and growth mechanism of the electro-polymerization of thiophene, show the presence both of instantaneous (2D and 3D) and of progressive contributions. The authors concluded that at higher concentrations of monomer, the prevalent growth mechanism is the progressive one, observing a diminution of the contribution of the instantaneous mechanism ${ }^{39}$.

We have plotted the number of thiophene units of the first layer of the film against the evolution of the simulation, till the same final time at the range of concentrations before mentioned (Figure 7b). Again, $\tau$ has been subtracted from all curves with the aim of having equivalent nucleation starting points. The plots correspond to a progressive nucleation, and as in the experimental study, a higher monomer concentration increases the appearance rate of nuclei with diffusion control.

The electro-polymerization process involves a reaction sequence in which each coupling step has to be activated by two species. The film-forming process needs two electrons per molecule, that is $2 \mathrm{~F} \cdot \mathrm{mol}^{-1}{ }^{40}$. Then, it is possible to simulate plots of the number of transferred electrons against the simulation time with theoretical expressions for the nucleation and growth mechanism. Plots of Figure $7 \mathrm{~b}$ were fitted using the progressive nucleation expression $(\mathrm{PN} 3 \mathrm{D})$ under diffusional control, $\mathrm{y}(\mathrm{t})=\mathrm{at}^{-0.5}\left[1-\exp \left(-\mathrm{bt}^{2}\right)\right]$. Figure 9 shows the adjusted profile obtained with a concentration of monomer of $0.5 \mathrm{M}$, presenting very good correlation, confirming the progressive nucleation process.

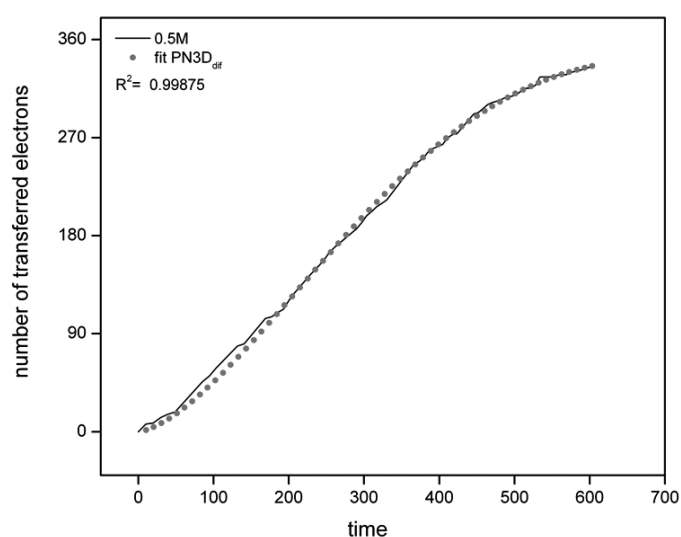

Figure 9: Number of electrons transferred to form the first layer of the film against the simulation time, and the simulation of this curve using PN3D under diffusional control

Figure 10 shows the coverage degree of the first layer of the deposit through the simulation time. All profiles reached a fixed value of approximately $25 \%$ of coverage degree due to the absence of diffusion after precipitation. As new 
thiophene oligomers are added to the conducting film, they block available sites at the bottom of the simulation box. With the programmed approximations, the first layer will never reach a complete coverage.

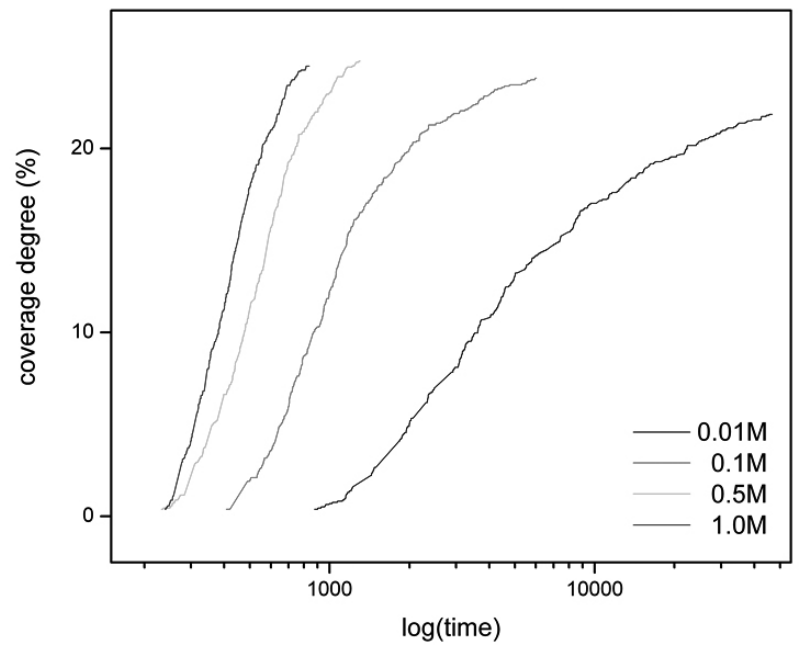

Figure 10: Coverage degree at $t_{f}$ of the first layer of the film against the simulation time (in logarithmic scale) at different concentrations

In spite of all simplifications applied to the stochastic model, we believe that the first results are quite encouraging; there was good agreement with experimental observations. The introduction of accurate rates or diffusion after precipitation will improve the current model and will provide more details to verify and confirm the nucleation and growth mechanism of conducting polymers.

\section{CONCLUSION}

With the aim of verifying experimental theories about the nucleation and growth process of the electrochemical conducting polymers synthesis, we have developed a new simulation scheme using stochastic methodologies. Like all simulation methods, our work demands a drastic simplification of the real system. Neither the solvent nor the supporting electrolyte was explicitly considered because of the size of the system. Despite all approximations the first results are quite favorable. Introduction of new considerations, like accurate diffusion coefficients or the diffusion of oligomers after precipitation, will improve the current model. New runs on other polymeric systems, other temperatures and rates will provide more details to corroborate the already proposed electro-polymerization mechanism.

\section{ACKNOWLEDGEMENTS}

M. B. C. is grateful to CONICYT for a Ph.D. fellowship and M.C.G. is grateful to CONICET and Alexander von Humboldt fellowship. The authors acknowledge to the financial support by FONDECYT grants $\mathrm{N}^{\circ} 1100055$ (M. A. del V.) W.S. thanks CONICET Argentina for continued support.

\section{REFERENCES}

1. Shirakawa, H.; Louis, E. J.; MacDiarmid, A. G.; Chiang, C. K.; Heeger, A. J., J. Chem. Soc., Chem. Commun. 1977, 578-580.

2. Groenendaal, L.; Meijer, E. W.; Vekemans, J. A., Nitrogen-Containing Oligomers. In Electronic Materials: The Oligomer Approach, Prof. Dr. K. Müllen, P. D. G. W., Ed. 2007; pp 235-275.

3. Glenis, S.; Benz, M.; LeGoff, E.; Schindler, J. L.; Kannewurf, C. R.; Kanatzidis, M. G., J. Am Chem. Soc. 1993, 115, 12519.

4. Kauffmann, T.; Lexy, H., Chemische Berichte 1981, 114, 3667.

5. Seixas de Melo, J.; Elisei, F.; Gartner, C.; Gaetano Aloisi, G.; Becker, R. S., J. Phys. Chem. A 2000, 104, 6907.

6. Bäuerle, P., Sulfur-Containing Oligomers. In Electronic Materials: The Oligomer Approach, Prof. Dr. K. Müllen, P. D. G. W., Ed. 2007; pp 105197.
7. McCullough, R. D., Adv. Mater. 1998, 10, 93

8. Kaminorz, Y.; Smela, E.; Johansson, T.; Brehmer, L.; Andersson, M. R.; Inganäs, O., Synth. Met. 2000, 113, 103-114.

9. Baeuerle, P.; Segelbacher, U.; Maier, A.; Mehring, M., J. Am. Chem. Soc. 1993, 115, 10217.

10. Barbarella, G.; Favaretto, L.; Sotgiu, G.; Zambianchi, P.; Bongini, A.; Arbizzani, C.; Mastragostino, M.; Anni, M.; Gigli, G.; Cingolano, R., J. Am. Chem. Soc. 2000, 122, 11971

11. Geiger, F.; Stoldt, M.; Schweizer, H.; Baeuerle, P.; Umbach, E., Adv Mater. 1993, 5, 922.

12. Ho, P. K. H.; Kim, J. S.; Burroughes, J. H.; Becker, H.; Li, S. F. Y.; Brown, T. M.; Cacialli, F.; Friend, R. H., Nature 2000, 404, 481.

13. Inganäs, O.; Berggren, M.; Andersson, M. R.; Gustafsson, G.; Hjertberg, T.; Wennerström, O.; Dyreklev, P.; Granström, M., Synth. Met. 1995, 71, 2121.

14. Kaminorz, Y.; Smela, E.; Inganäs, O.; Brehmer, L., Adv. Mater. 1998, 10, 765-769.

15. Pinner, D. J.; Friend, R. H.; Tessler, N., Appl. Phys. Lett. 2000, 76, 1137.

16. Ding, L.; Jonforsen, M.; Roman, L. S.; Andersson, M. R.; Inganäs, O., Synth. Met. 2000, 110, 113.

17. Granström, M.; Petrisch, K.; Arias, A. C.; Lux, A.; Andersson, M. R.; Friend, R. H., Nature 1998, 395, 257.

18. Hoppe, H.; Sariciftci, N. S., J. Mater. Res. 2004, 19, 1924.

19. Otsubo, T.; Aso, Y.; Takimiya, K., Bull. Chem. Soc. Jpn. 2001, 74, 1789.

20. Salomon, A.; Cahen, D.; Lindsay, S.; Tomfohr, J.; Engelkes, V. B.; Frisbie, C. D., Adv. Mater. 2003, 15, 1881.

21. Dodabalapur, A.; Katz, H. E.; Torsi, L.; Haddon, R. C., Science $\mathbf{1 9 9 5}$ 269,1560 .

22. Garnier, F.; Hajlaoui, R.; Yassar, A.; Srivastava, P., Science 1994, 265 , 1684.

23. Garnier, F., Chem. Phys. 1998, 227, 253.

24. Horowitz, G.; Peng, X.; Fichou, D.; Garnier, F., J. Appl. Phys. 1990, 67, 528.

25. Matin, R. E.; Diederich, F., Angew. Chem., Int. Ed. 1999, 38, 1350

26. Robertson, N.; McGowan, C. A., Chem. Soc. Rev. 2003, 32, 96.

27. Würthner, F., Angew. Chem., Int. Ed. Engl. 2001, 40, 1037.

28. Roncali, J., Chem. Rev. 1992, 92, 711-738.

29. Genies, E. M.; Bidan, G.; Diaz, A. F., J. Electroanal. Chem. 1983, 149 , 101-113.

30. Dai, Y.; Blaisten-Barojas, E., J. Chem. Phys. 2010, 133, 034905-7.

31. Mariscal, M.; Leiva, E.; Pötting, K.; Schmickler, W., Applied Physics A. Materials Science \& Processing 2007, 87, 385-389.

32. Schmickler, W.; Pötting, K.; Mariscal, M., Chem. Phys. 2006, 320, 149154.

33. Angélica del Valle, M.; Díaz, F. R.; Bodini, M. E.; Alfonso, G.; Soto, G. M.; Borrego, E. D., Polymer International 2005, 54, 526-532.

34. Almenninger, A.; Bastiansen, O.; Suendas, P., Acta Chem. Scand. 1958 , 12,1671 .

35. Lang, P.; Chao, F.; Costa, M.; Garnier, F., Polymer 1987, 28, 668-674.

36. Heinze, J.; Frontana-Uribe, B. A.; Ludwigs, S., Chem. Rev. 2010, 110, 4724-4771.

37. del Valle, M. A.; Diaz, F. R.; Bodini, M. E.; Alfonso, G.; Soto, G. M.; Borrego, E. D., Polymer International 2005, 54, 526-532.

38. del Valle, M. A.; Cury, P.; Schrebler, R., Electrochim. Acta 2002, 48, 397-405.

39. Schrebler, R.; Grez, P.; Cury, P.; Veas, C.; Merino, M.; Gomez, H.; Cordova, R.; del Valle, M. A., J. Electroanal. Chem. 1997, 430, 77-90.

40. Diaz, A. F.; Castillo, J. I.; Logan, J. A.; Lee, W.-Y., J. Electroanal. Chem. 1981, 129, 115-132.

41. Press, W.; Teukolsky, S.;Vetterling, W.; Flannery, B., Numerical Recepies in Fortran 77, The Art of Scientific Computing, Cambridge University Press, Cambridge, 1986-1992.

42. del Valle, M. A.; Gacitúa, M.; Canales, L. I.; Díaz, F.R., J. Ch. Chem. Soc. $\mathbf{2 0 0 9}, 54,260$ 